\title{
Research on Fuel Economy Aimed Coordination Control Strategy for the Dual-mode Hybrid System under Dynamic Conditions
}

\author{
Yu Zhao, Changlu Zhao,Ying Huang* and Long Yang \\ School of Mechanical Engineering, Beijing Institute of Technology, Beijing, China \\ ${ }^{*}$ Corresponding author
}

\begin{abstract}
A simulation of dynamic conditions was carried out based on the energy management strategy of "multi-objective power flow optimization based on engine optimal fuel economy curve". In dynamic conditions, without considering the response time of the adjustment of engine speed and engine torque, the motor torque loads too fast, the speed control of the engine and the torque control of the motors are not coordinated, which makes the accuracy and stability of dynamic condition control poor. In order to solve the incoordination problem in dynamic conditions, the coordination control strategy of "The target motor torque signal is subjected to first-order hysteresis treatment + The target engine speed signal is subjected to slope control treatment" was proposed, and the simulation research was carried out. The simulation results show that the coordination control strategy can effectively improve the tracking of the optimal fuel economy curve, improve the fuel economy performance of the engine, and improve the accuracy and stability of the dynamic condition control.
\end{abstract}

Keywords-dual-mode; energy management; coordination control; fuel economy performance; accuracy and stability

\section{INTRODUCTION}

A hybrid system combines the advantages of both mechanical driving and electric driving to improve the fuel economy performance and emission performance of the system, mitigate energy crisis problem and air pollution problem [1]. A dual-mode hybrid system is an important form of hybrid systems. Under the condition that the engine speed is constant, the vehicle speed can monotonically rise or monotonically reduce with the motor speed. When the working state of the control components in the coupling mechanism is changed, the system can get into another mode from one mode, the motor speed changes in the opposite direction, and the output shaft speed of the coupling mechanism changes continuously to realize continuous speed change [2]. A dual-mode hybrid system has the advantages of large driving power and wide speed range, which can reduce the power requirements of the motors and shows a good application prospect.

The dual-mode hybrid system is more suitable for heavy vehicles, whose power allocation control and coordination control are more complex. First, in steady-state conditions, according to the energy management strategy, the engine and the motors are allocated power. Whether the engine and the motors can track their target power curve or not is very critical. Secondly, in dynamic conditions, whether the engine and the motors can coordinate with each other to ensure the accuracy and stability of adjustment process is equally important, while meeting the power requirements of the system. In order to solve the problem of energy management and power allocation in steady-state conditions, domestic and foreign scholars have done much research [3-6]. In order to solve the problem of coordination control of the engine and the motors in dynamic conditions, domestic and foreign scholars have also done some research [7-9]. However, most of the coordination control research focus on how to reduce the torque ripple in the mode switching process. There is little research on the accuracy and stability of coordination control to meet the power requirements of the system, without the working state of the control components changing.

The energy management strategy based on the optimal fuel economy curve was applied to the dual-mode hybrid system. The simulation results show that the operating points of the engine are far from the optimal fuel economy curve, and the adjustment process is imprecise and unstable in dynamic conditions. In this paper, in order to solve the incoordination problem in dynamic conditions, the coordination control strategy of "The target motor torque signal is subjected to firstorder hysteresis treatment + The target engine speed signal is subjected to slope control treatment" was proposed and verified by simulation. This strategy takes into account the response time of the adjustment process of the engine in dynamic conditions, the loading of motor torque slows down, and the target engine speed and target engine torque are closer to the optimal fuel economy curve. The simulation results show that the coordination control strategy can improve the fuel economy performance of the engine and improve the accuracy and stability of the dynamic process regulation.

\section{ANALYSIS OF SYSTEM CHARACTERISTICS}

\section{A. Analysis of System Structure Characteristics}

The structure of the dual-mode hybrid system is shown in Figure 1 . The system consists of the engine, the motor $A$, the motor $\mathrm{B}$, the batteries, the power coupling mechanism, the power allocation unit, the front transmission, the rear transmission and other components. The energy of the engine is transmitted by the mechanical power flow and the electric power flow to meet the requirements for high power and high torque of heavy vehicles.

The structure of the power coupling mechanism is shown in Figure 2. The power coupling mechanism is used to achieve shunt and confluence of mechanical power. The power coupling mechanism consists of three planetary rows, one brake Z2, and 
one clutch $\mathrm{C} 1$. When the working state of the brake or clutch (combination or separation) is changed, the mode of the hybrid system changes accordingly.

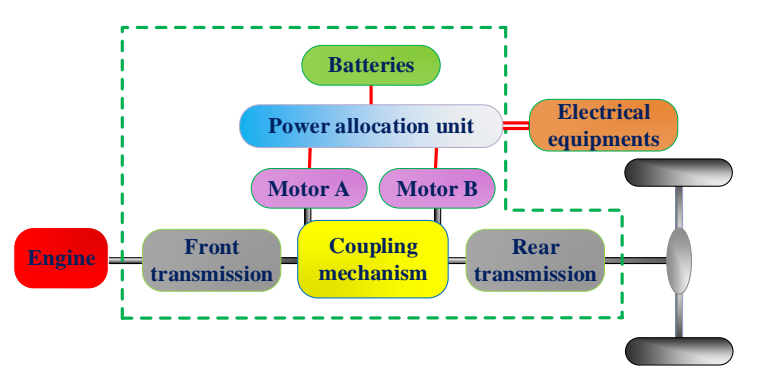

FIGURE I. STRUCTURE OF THE DUAL -MODE HYBRID SYSTEM

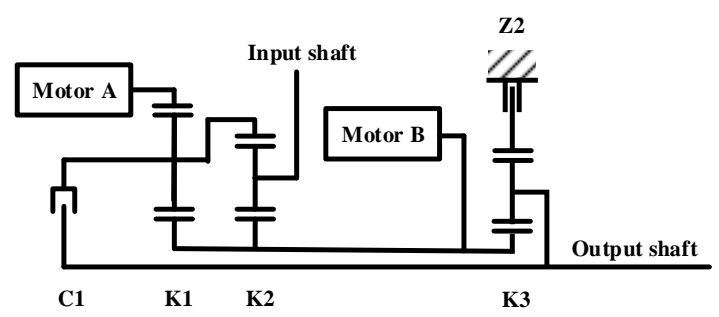

FIGURE II. STRUCTURE OF THE POWER COUPLING MECHANISM

The power allocation unit is used to achieve shunt and confluence of electric power. The front transmission is used to achieve the growth of the engine output shaft speed to match the motor speed. The rear transmission is used to realize the change of the range of the power coupling mechanism output shaft speed. The engine is the power source of the hybrid system, whose power flow is divided into two parts. One part is transmitted in the form of mechanical power; the other part drives the generator to generate electricity and then is transmitted in the form of electric power. The batteries are used to balance the power deviation between the generator, the motor, and electrical equipment. If the power of the generator is greater than the sum of the power of the motor and electrical equipment, the excess electric power flows to the batteries, otherwise, the batteries compensate for insufficient electric power. Finally, the mechanical power from the engine and the mechanical power from the motor are coupled by the power coupling mechanism and then transmitted to the wheel via the rear transmission to drive the vehicle.

\section{B. Analysis of Speed Characteristics and Torque Characteristics of the System}

Because the engine, the motor $\mathrm{A}$, and the motor $\mathrm{B}$ are connected to the different positions of the planetary rows of the power coupling mechanism, there are specific coupling constraints between the speed and torque of the engine, the motor A, the motor $\mathrm{B}$, and the coupling mechanism output shaft. When the working state of the brake or clutch is changed, the position of the engine, the motor $\mathrm{A}$, and the motor $\mathrm{B}$ connected to the planetary rows will change accordingly. Therefore, in the EVT1 mode (the brake $\mathrm{Z} 2$ is engaged, the clutch $\mathrm{C} 1$ is disconnected) and the EVT2 mode (the clutch C1 is engaged, and the brake $\mathrm{Z2}$ is disconnected), the speed characteristics and torque characteristics of the system are different.
The speed characteristics and torque characteristics in the EVT1 mode are as follows:

$$
\begin{gathered}
{\left[\begin{array}{c}
n_{\mathrm{a}} \\
n_{b}
\end{array}\right]=\left[\begin{array}{cc}
\frac{\left(1+k_{1}\right)\left(1+k_{2}\right)}{k_{1} k_{2}}-\frac{\left(1+k_{1}+k_{2}\right)\left(1+k_{3}\right)}{k_{1} k_{2}} \\
1+k_{3}
\end{array}\right]\left[\begin{array}{l}
n_{i} \\
n_{o}
\end{array}\right],} \\
{\left[\begin{array}{c}
T_{\mathrm{a}} \\
T_{\mathrm{b}}
\end{array}\right]=\left[\begin{array}{ll}
-\frac{k_{1} k_{2}}{\left(1+k_{1}\right)\left(1+k_{2}\right)} & 0 \\
-\frac{1+k_{1}+k_{2}}{\left(1+k_{1}\right)\left(1+k_{2}\right)} & \frac{1}{1+k_{3}}
\end{array}\right]\left[\begin{array}{l}
T_{\mathrm{i}} \\
T_{\mathrm{o}}
\end{array}\right] .}
\end{gathered}
$$

The speed characteristics and torque characteristics in the EVT2 mode are as follows:

$$
\begin{gathered}
{\left[\begin{array}{l}
n_{\mathrm{a}} \\
n_{\mathrm{b}}
\end{array}\right]=\left[\begin{array}{cc}
-\frac{1+k_{2}}{k_{1}} \frac{\left(1+k_{1}+k_{2}\right)}{k_{1}} \\
1+k_{2} & -k_{2}
\end{array}\right]\left[\begin{array}{l}
n_{i} \\
n_{o}
\end{array}\right]} \\
{\left[\begin{array}{l}
T_{\mathrm{a}} \\
T_{\mathrm{b}}
\end{array}\right]=\left[\begin{array}{ll}
-\frac{k_{1} k_{2}}{\left(1+k_{1}\right)\left(1+k_{2}\right)} & \frac{k_{1}}{1+k_{1}} \\
-\frac{1+k_{1}+k_{2}}{\left(1+k_{1}\right)\left(1+k_{2}\right)} & \frac{1}{1+k_{1}}
\end{array}\right]\left[\begin{array}{l}
T_{\mathrm{i}} \\
T_{\mathrm{o}}
\end{array}\right] .}
\end{gathered}
$$

Where $n_{\mathrm{a}}$ is the speed of the motor $\mathrm{A}, n_{\mathrm{b}}$ is the speed of the motor $\mathrm{B}, n_{\mathrm{i}}$ is the speed of the power coupling mechanism input shaft, $n_{\mathrm{o}}$ is the speed of the power coupling mechanism output shaft. $T_{\mathrm{a}}$ is the torque of the motor $\mathrm{A}, T_{\mathrm{b}}$ is the torque of the motor $\mathrm{B}, T_{\mathrm{i}}$ is the torque of the power coupling mechanism input shaft, $T_{0}$ is the torque of the power coupling mechanism output shaft. $k_{1}, k_{2}$ and $k_{3}$ are the characteristic parameters of the planetary rows.

\section{SimUlATION AND ANALYSIS OF DyNAMIC CONDITIONS}

\section{A. Energy Management Strategy}

The process of the adopted energy management strategy [10] is shown in Figure 3.

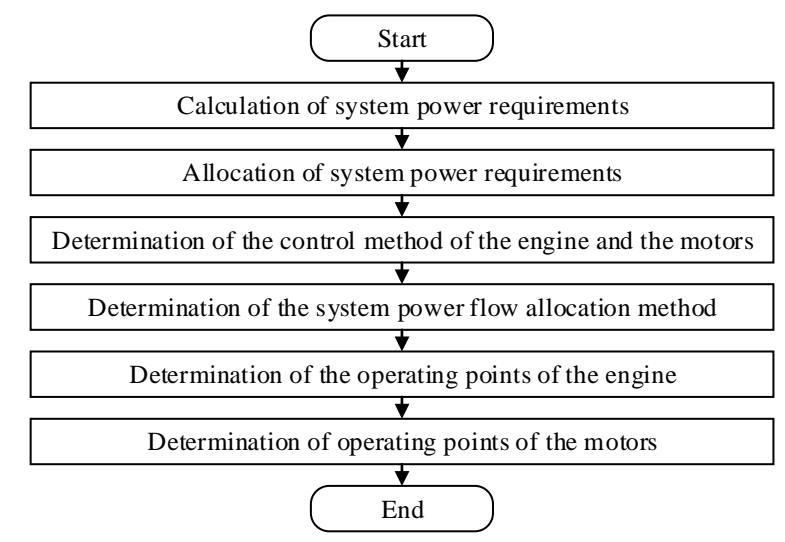

FIGURE III. PROCESS OF THE ADOPTED ENERGY MANAGEMENT STRATEGY 


\section{1) Calculation of system power requirements}

The system power requirements include driving power requirements and electric power requirements of the vehicle. The driving power requirements are linear with the accelerator pedal opening generally. The electric power requirements include the power requirements of the electrical equipment and the power requirements of the batteries.

\section{2) Allocation of system power requirements}

Limited to the space constraints of heavy vehicles, the capacity of the selected batteries is low. The principle of using the batteries is to try to maintain SOC the constant value of initial SOC, to avoid overcharge and over discharge. In the case of rapid acceleration or braking, the batteries are allowed to discharge or charge. Therefore, in general, the driving power requirements and the electric power requirements of the vehicle are all provided by the engine.

3) Determination of the control method of the engine and the motors

In the dual-mode hybrid system, the engine uses the speed control method; the motors use the torque control method. According to the analysis of the torque characteristics of the system, the torque of the engine can be adjusted by the torque of the engine load, that is, by the torque of the motors. According to the analysis of the speed characteristics of the system, the speed of the motors can be determined by the speed of the engine and vehicle speed.

4) Determination of the system power flow allocation method

In this paper, "multi-objective power flow optimization based on the optimal fuel economy curve of the engine" method was adopted. That how to allocate the power flow of the system directly affect electric power supply performance, driving performance and fuel economy performance of the system. In order to improve the fuel economy performance of the system, the engine is forced to keep track of the optimal fuel economy curve. On this basis, the priority of the system optimization objectives is determined, then the system power flow is allocated and optimized. The electrical equipment of the vehicle directly affects the safety performance of the vehicle, so the priority of the electric power supply performance is higher than the priority of driving performance.

\section{5) Determination of operating points of the engine}

The power requirements of the engine are determined according to the driving power requirements and the electric power requirements of the system. The target speed of the engine is determined according to the optimal fuel economy curve of the engine. The target torque of the engine is determined by the relationship among power, speed, and torque.

6) Determination of the operating points of the motors

From the analysis of speed characteristics and torque characteristics of the system, it can be seen that the speed of the motors is determined according to the input shaft speed and the output shaft speed of the power coupling mechanism, that is, determined by the engine speed and vehicle speed. The motors are the engine load, and the motor torque needs to be controlled to adjust the engine torque through the torque coupling constraint relationship. On this basis, according to the priority of multi-objective power flow optimization objectives, the feasible domain range of the motor torque is gradually reduced, the system power flow is optimized, and finally, the torque of the motors is determined.

\section{B. Simulation of Dynamic Conditions}

A simulation was carried out based on this energy management strategy to test control effects and detect control problems.

\section{1) Design of the simulation condition}

The electric power requirements of the electrical equipment are temporarily not considered. The vehicle drives straight without turning, and there are obvious dynamic processes taking place in the EVT1 mode and the EVT2 mode.

The accelerator pedal opening given in the simulation condition is shown in Figure 4. $0-15 \mathrm{~s}$, the accelerator pedal opening is $0.25,15-40 \mathrm{~s}$, the accelerator pedal opening is 0.5 , 40 $65 \mathrm{~s}$, the accelerator pedal opening is 0.75 . There are two obvious dynamic processes occurring at $15 \mathrm{~s}$ and $40 \mathrm{~s}$.

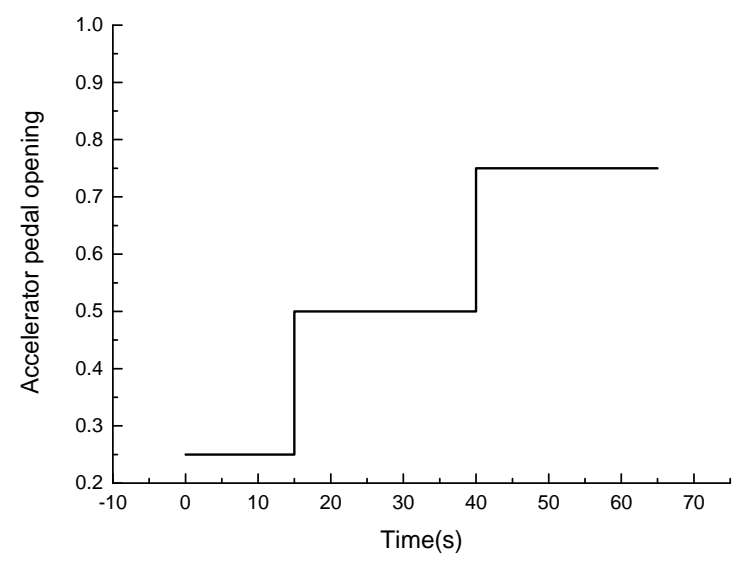

FIGURE IV. ACCELERATOR PEDAL OPENING

2) Analysis of the simulation results

The vehicle speed and EVT mode status are shown in Figure 5. The system is in the EVT1 mode as EVT mode status value equals to 1, the system is in the mode switching process as EVT mode status value equals to 2 , the system is in the EVT2 mode as EVT mode status value equals to 3 . At 5.20 s, the engine got into conventional condition, the system got into the EVT1 mode, and the vehicle began to move forward. In the case that the power of the engine (proportional to the accelerator pedal opening) was constant, the driving torque and the acceleration of the vehicle were getting smaller and smaller due to the increasing vehicle speed. At $15 \mathrm{~s}$ and 40 s, the power of the engine significantly increased, the acceleration of the vehicle significantly increased accordingly. The mode switching speed is $32 \mathrm{~km} / \mathrm{h}$. At 27.00s-27.05s, the mode switched, and the vehicle speed slightly reduced. After the system had got into the EVT2 mode, the vehicle continued to accelerate. The first obvious dynamic process took place in the EVT1 mode, and the second obvious dynamic process took place in the EVT2 mode.

The engine speed, engine torque, and engine power are shown in Figure 6. The speed of the engine is controlled by the closed loop PID algorithm, and the actual speed of the engine 
can track the target speed of the engine well. The overshoot in the dynamic adjustment process is small and the adjustment time is short. The torque of the engine is adjusted by the torque of the motors, which is determined in real time according to the power flow optimization objectives of the system. In the steady-state processes, the actual torque of the engine can roughly track the target torque of the engine. In the dynamic processes occurring at $15 \mathrm{~s}$ and $40 \mathrm{~s}$, the actual torque of the engine deviates greatly from the target torque of the engine. (The torque ripple control in the mode switching process occurring at 27s involves the control of the control components such as clutches and brakes, not covered by the scope of this paper.)
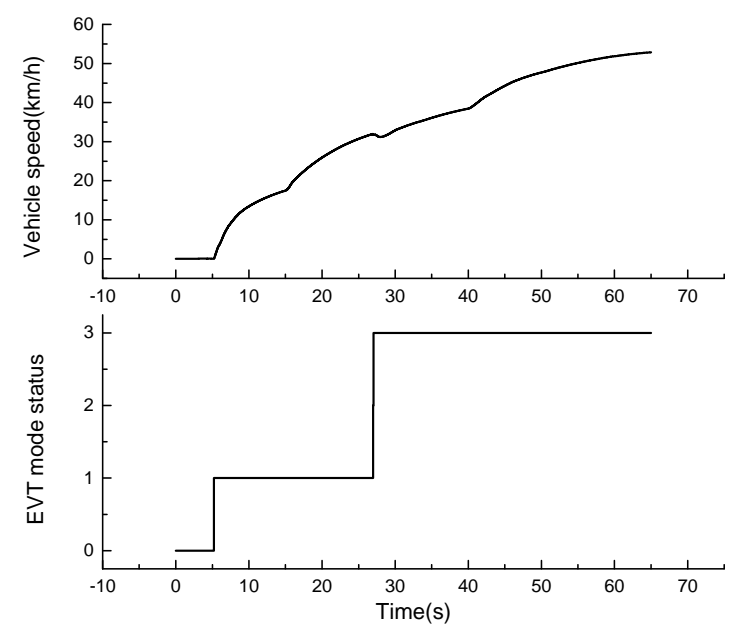

FIGURE V. VEHICLE SPEED AND EVT MODE STATUS
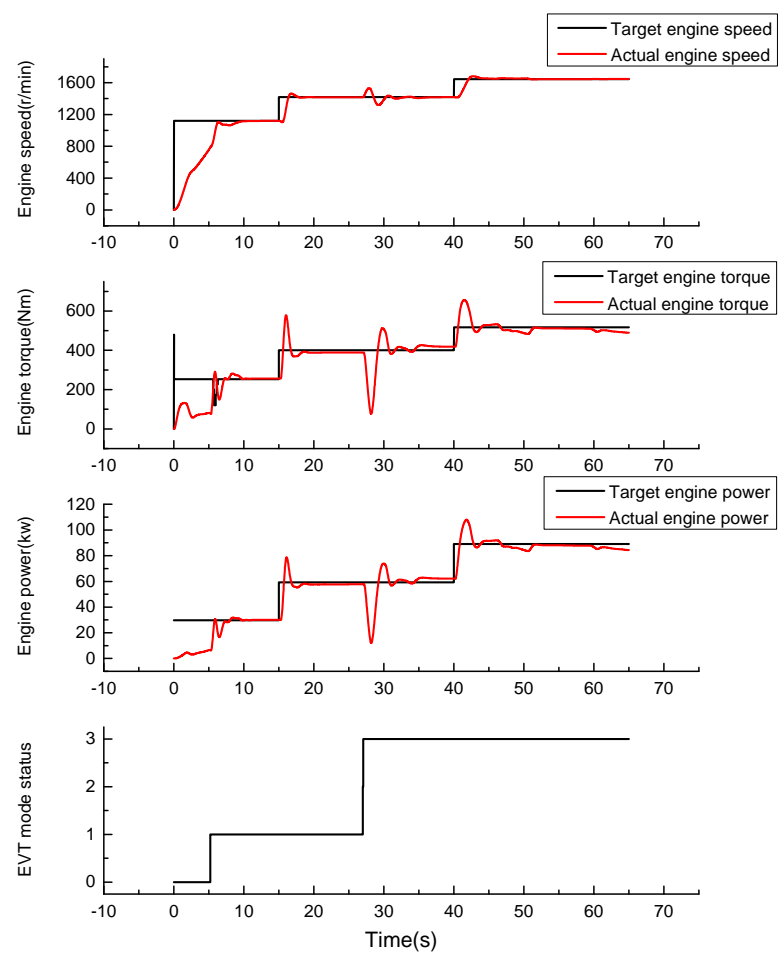

FIGURE VI. ENGINE SPEED, ENGINE TORQUE, ENGINE POWER
The first obvious dynamic process was taken as an example to analyze the control of the engine in dynamic conditions. The local engine speed and local engine torque are shown in Figure 7. At $15 \mathrm{~s}$, the target engine speed increased significantly, but the actual engine speed reduced first, which is due to that the response time of the engine torque is far greater than the response time of the motor torque. When the target engine torque is greatly raised, the torque of the engine load can be momentarily adjusted to the value of target engine torque, but it needs some time that the actual engine torque increases to the value of target engine torque, so the actual engine speed first reduced. The abnormal state of the engine speed will lead that the vehicle cannot respond to the acceleration intention of the driver in time, and the vehicle speed cannot rise in time. When the engine speed is low, the engine will even turn off and stop the vehicle. In order to complete the speed control process, the actual engine torque will rise to higher than the engine load torque. The engine load torque can be momentarily adjusted to the value of the target engine torque, which makes the maximum of the actual torque of the engine will be far from the value of the target torque. The abnormal state of engine torque will lead that the engine operating points deviate from the optimal fuel economy curve, which deteriorates the fuel economy performance of the system. The minimum of actual engine torque will deviate from the value of the target torque under the condition that the target engine speed is drastically reduced, which may cause the engine to turn off and stop the vehicle.

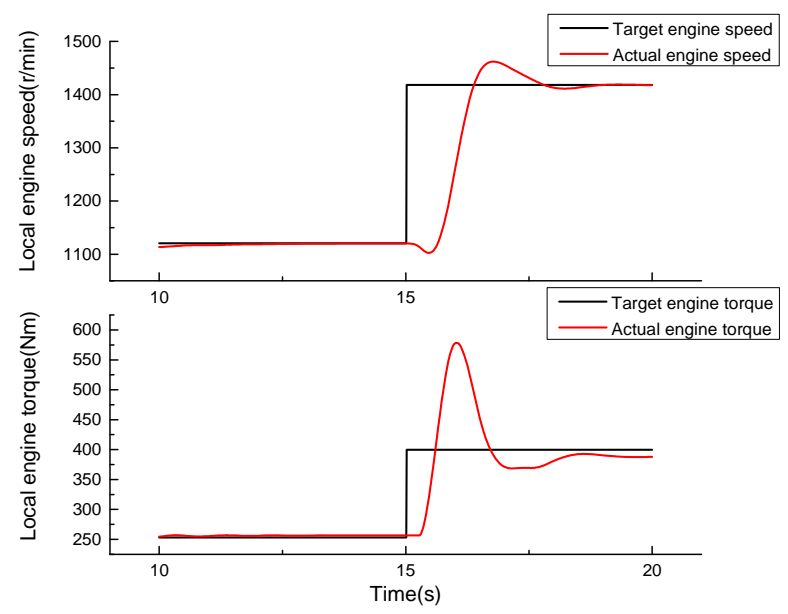

FIGURE VII. LOCAL ENGINE SPEED AND LOCAL ENGINE TORQUE

The speed and power of the local engine operating points are shown in Figure 8. In the dynamic process, the engine cannot track the optimal fuel economy curve accurately, and the fuel economy performance of the system is very poor. This is due to that the difference of the response time of the engine torque and the response time of the motor torque is not considered. It is impossible to instantly complete the adjustment process of the engine speed and torque changes. The loading of the motor torque is too fast, which leads that the speed control of the engine and the torque control of the motors are not coordinated in the dynamic process. The engine operating points are far from the optimal fuel economy curve, the working state of the engine is abnormal, and the accuracy and stability of the dynamic process adjustment are poor. 


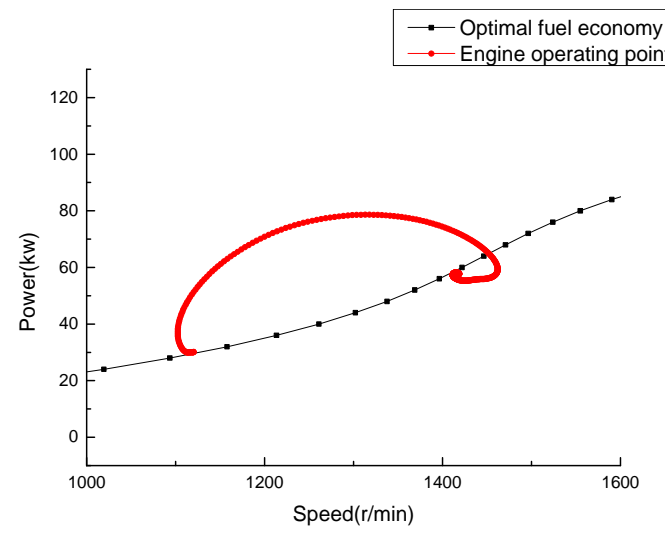

FIGURE VIII. SPEED AND POWER OF THE LOCAL ENGINE OPERATING POINTS

The motor speed, motor torque, motor power are shown in Figure 9. In the EVT1 mode, the motor A speed is positively correlated with the engine speed and negatively correlated with the vehicle speed. Therefore, when the engine speed was constant, the speed of the motor A reduced with the increase of the vehicle speed, and the motor A speed greatly increased when the engine speed greatly increased. The motor B speed is proportional to the vehicle speed. When the vehicle speed increased, the motor B speed also increased. The analysis of motor speed in the EVT2 mode is similar to the analysis in the EVT1 mode, and the motor speed is also in accordance with the coupling constraints in the EVT2 mode. After the mode was switched, the motor speed changed in the opposite direction. In the EVT1 mode, the motor A is the load of the engine. In steady - state conditions, the value of the engine load torque calculated by the motor $A$ torque is close to the actual engine torque. In the EVT2 mode, the motor A and the motor B are both the load of the engine. In steady - state conditions, the value of the engine load torque calculated by the motor A torque and the motor B torque is close to the actual engine torque. The absolute values of the electric power of the motor $A$ and the electric power of the motor B are basically the same, and the signs are opposite. The electric power deviation is regulated by the batteries, and the electrical power of the system is balanced. In the EVT1 mode, the power of the motor $A$ is basically negative, and the motor $A$ is the generator; the power of the motor $\mathrm{B}$ is basically positive, the motor $\mathrm{B}$ is the motor. In the EVT2 mode, the power of the motor $\mathrm{A}$ is basically positive, and the motor $\mathrm{A}$ is the motor; the power of the motor B is basically negative, the motor B is the generator.

The batteries SOC, batteries current, batteries voltage, batteries power are shown in Figure 10. The batteries SOC is always maintained near the initial SOC 60 , and the batteries voltage is always maintained near the initial voltage $391 \mathrm{~V}$. The batteries current are within 20A, and the batteries power is within $10 \mathrm{kw}$. The batteries are in good condition, no overcharge and over discharge.
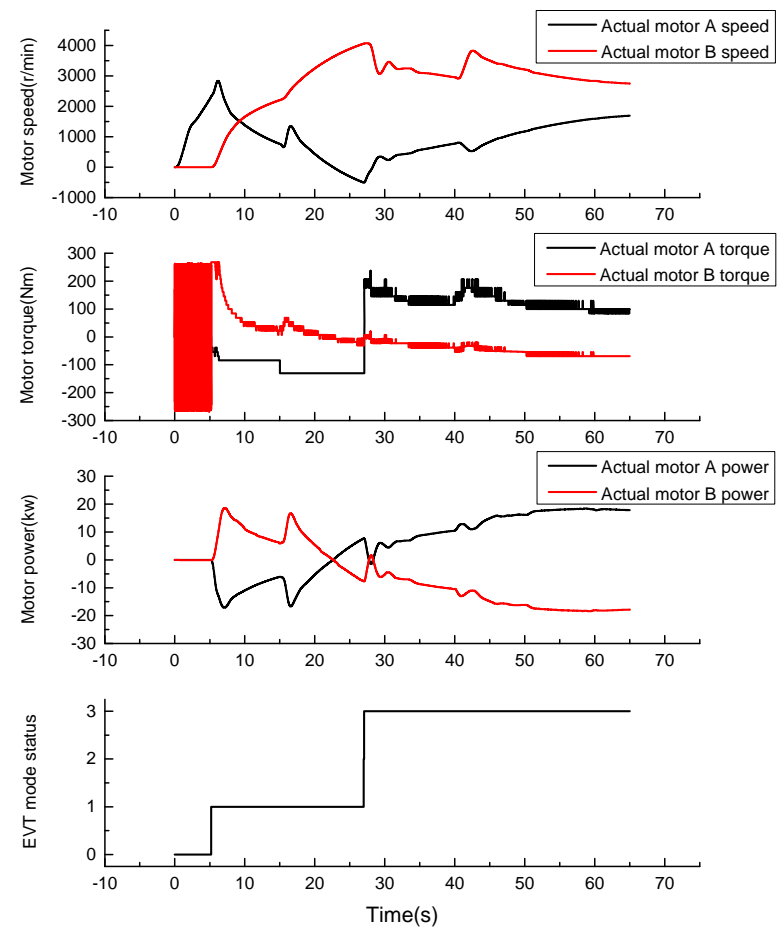

FIGURE IX. MOTOR SPEED, MOTOR TORQUE, MOTOR POWER
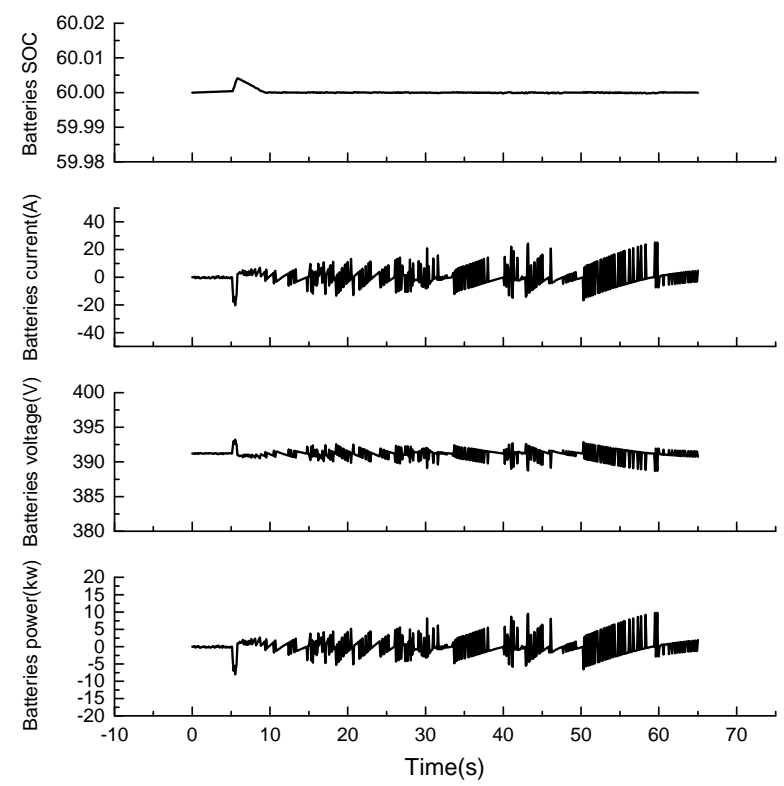

FIGURE X. BATTERIES SOC, BATTERIES CURRENT, BATTERIES VOLTAGE, BATTERIES POWER

\section{SimUlation AND ANALYSIS OF COORDINATION CONTROL STRATEGY FOR THE ENGINE AND THE MOTORS IN DYNAMIC CONDITIONS}

The simulation results of Chapter 3 show that the engine, the motors and the batteries are in good working state and the control effect is very good under the steady - state condition, but the control effect is not ideal under the dynamic condition. The engine operating points deviate from the optimal fuel economy curve greatly, and the accuracy and stability of the dynamic 
process control are poor. In order to solve the problem that the regulation of engine speed and engine torque needs some time and the loading of motor torque is too fast in the dynamic process, the coordination control strategy of "The target motor torque signal is subjected to first-order hysteresis treatment + The target engine speed signal is subjected to slope control treatment" was proposed.

\section{A. The target motor torque signal is subjected to first-order hysteresis treatment}

"The target motor torque signal is subjected to first-order hysteresis treatment" method can slow the loading of motor torque, and the engine load torque can be loaded slowly rather than be instantaneously loaded to the value of target torque. When the time constant $\mathrm{T}$ is different, the motor $\mathrm{A}$ torque in the dynamic process occurring at $15 \mathrm{~s}$ is shown in Figure 11 . The greater the $\mathrm{T}$, the slower the loading of motor torque.

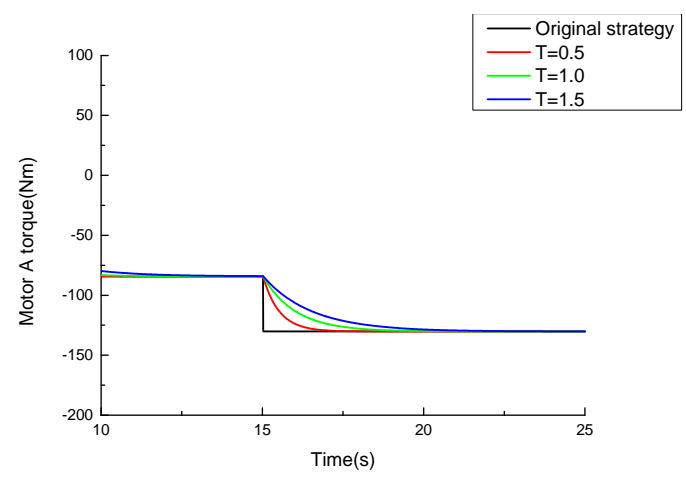

FIGURE XI. MOTOR A TORQUE

Similarly, the first obvious dynamic process was taken as an example to analyze the control of the engine in the dynamic process. The engine speed and engine torque when the $\mathrm{T}$ is different are shown in Figure 12. After the coordination control strategy is implemented, the loading of motor torque slows down. The loading process of the engine load torque also needs some time, the abnormal state of engine speed that the target engine speed increases but the actual engine speed first reduces is improved. At the same time, the maximum of actual engine torque does not need to be so large to complete the engine speed control process, the abnormal state of engine torque is improved.

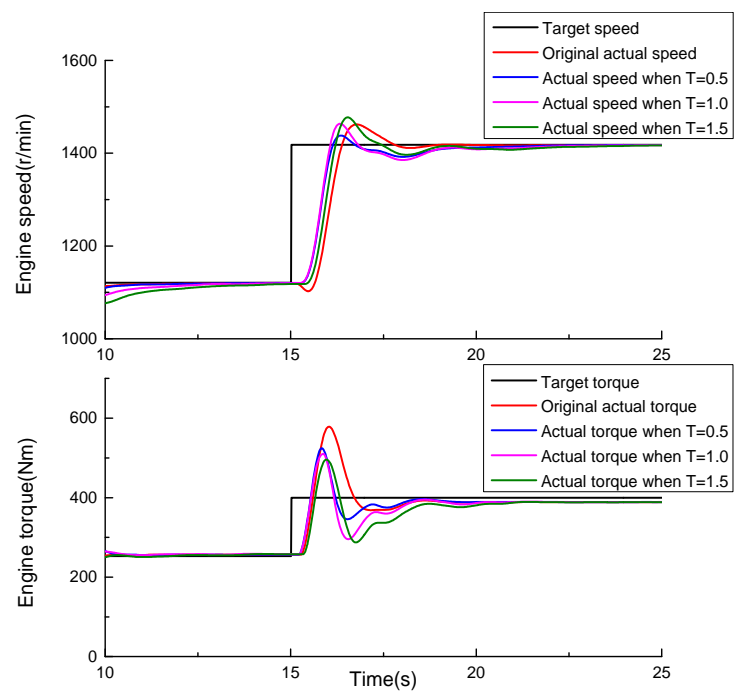

FIGURE XII. ENGINE SPEED AND ENGINE TORQUE WHEN THE T IS DIFFERENT

The larger the $\mathrm{T}$, the slower the loading of the engine load, and the shorter the speed control process. In order to maintain the engine speed at the value of target speed, the engine torque will be adjusted to the vicinity of the value of engine load torque, and then changes with the engine load torque to the value of target engine torque. If the $\mathrm{T}$ is too large, the engine load torque will be too small when the engine completes the speed control process, which will make the engine operating points deviate from the optimal fuel economy curve.

The engine speed and engine power of the engine operating points when the $\mathrm{T}$ is different are shown in Figure 13. After the coordination control strategy is implemented, the engine operating points are closer to the optimal fuel economy curve. However, if the $\mathrm{T}$ value is too large, the loading of motor torque is too slow, the fuel economy of engine in the latter part of the dynamic process will be worse.

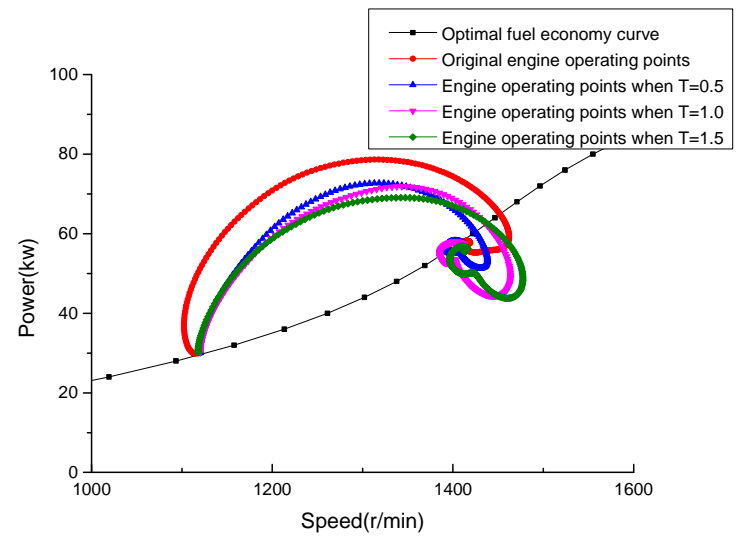

FIGURE XIII. ENGINE SPEED AND ENGINE POWER OF THE ENGINE OPERATING POINTS 
B. The target motor torque signal is subjected to first-order hysteresis treatment + The target engine speed signal is subjected to slope control treatment

The method of "The target motor torque signal is subjected to first-order hysteresis treatment" can improve the fuel economy performance of the engine in the dynamic process regulation and improve the accuracy and stability of the dynamic process regulation. However, the improvement effect is still limited. Compared to the engine speed adjustment process, the engine torque adjustment process is still faster. "The target engine speed signal is subjected to slope control treatment" method can limit the change rate of target engine speed, the deviation of actual engine speed and target engine speed becomes smaller than before, and the adjustment process of engine torque slows down.

In different dynamic conditions, the time required for the engine to complete the speed control process is different. The time constant selection of the first method is also different, which requires a lot of calibration work. Taking the time constant $\mathrm{T}=1.0$ as an example, the control effect of the coordination control strategy of "The target motor torque signal is subjected to first-order hysteresis treatment + The target engine speed signal is subjected to slope control treatment" was analyzed.

When the $\mathrm{T}=1.0$ and the slope is different, the engine speed and engine torque are shown in Figure 14. The smaller the slope, the slower the adjustment process, and the smaller the maximum of actual engine torque.

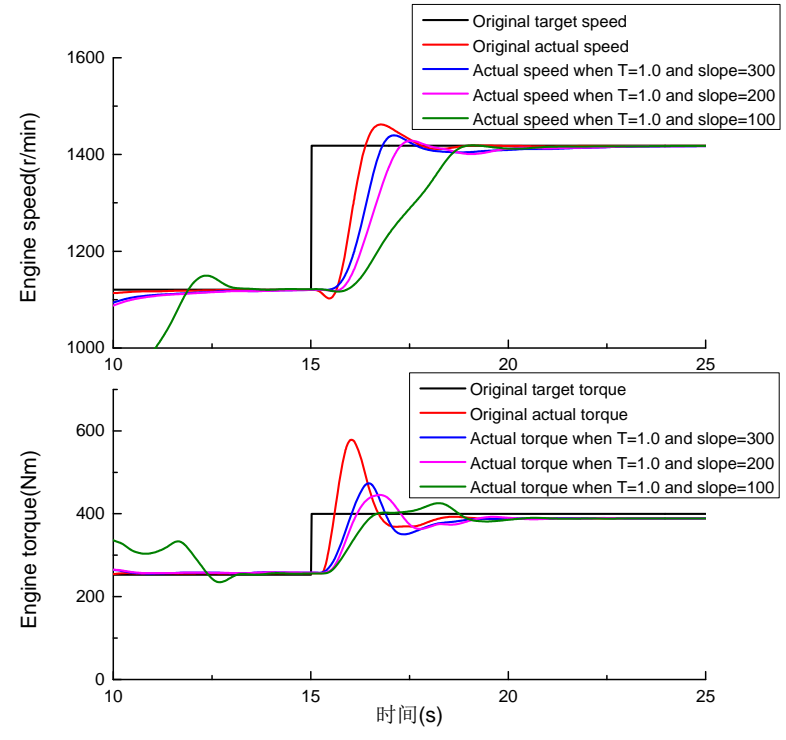

FIGURE XIV. ENGINE SPEED AND ENGINE TORQUE WHEN THE $\mathrm{T}=1.0$ AND THE SLOPE IS DIFFERENT

When the $\mathrm{T}=1.0$ and the slope is different, the speed and the power of the engine operating points are shown in Figure 15. The smaller the slope is, the closer the engine operating points are to the optimal fuel economy curve, the better the fuel economy performance of the engine is, the better the accuracy and stability of the dynamic process control are. Compared to the single application of the first method, the control effect of
"The target motor torque signal is subjected to first-order hysteresis treatment + The target engine speed signal is subjected to slope control treatment" method is better. If the slope is too large, the dynamic process adjustment time will extend, and the power output will be affected. Under different dynamic conditions, the change range of the target engine speed is different, and the selection of the slope is correspondingly different, which requires a lot of calibration work.

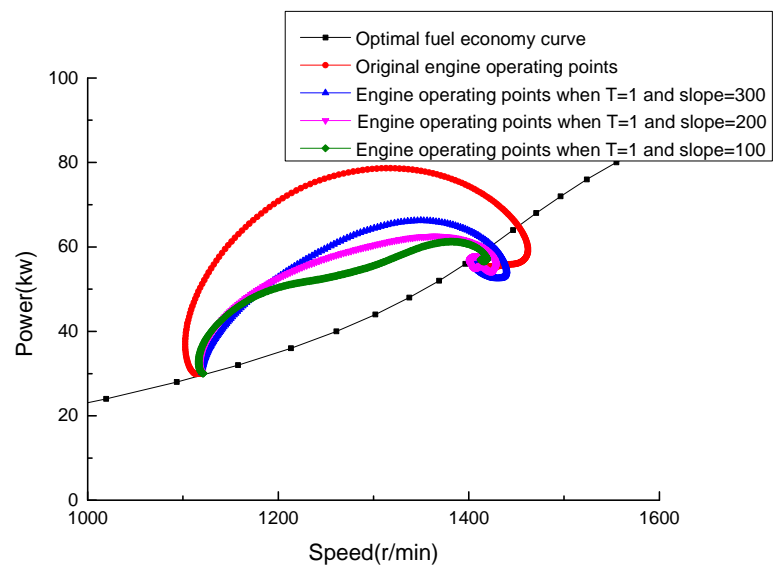

FIGURE XV. SPEED AND POWER OF THE ENGINE OPERATING POINTS WHEN THE T=1.0 AND THE SLOPE IS DIFFERENT

From the above analysis, we can see that the coordination control strategy can improve the tracking of the optimal fuel economy curve in dynamic process to a large extent, improve the fuel economy performance of the engine, improve the accuracy and stability of dynamic process control. The T and the slope need to be calibrated under different conditions.

\section{CONCLUSIONS}

(1) A simulation of dynamic conditions was carried out based on the energy management strategy of "multi-objective power flow optimization based on engine optimal fuel economy curve". The simulation results show that the control effect is good in the steady - state condition and is poor in the dynamic condition.

(2)In the dynamic condition, the engine operating points deviate from the optimal fuel economy curve greatly, and the accuracy and stability of dynamic condition control are poor. The reason why the control effect is poor in the dynamic coordination was analyzed in detail. The control strategy does not take into account the response time of the adjustment of engine speed and engine torque, the loading of motor torque is too fast, the speed control of the engine and the torque control of the motors are not coordinated.

(3)In order to solve the incoordination problem that the speed control of the engine and the torque control of the motors are not coordinated in the dynamic condition, the coordination control strategy of "The target motor torque signal is subjected to firstorder hysteresis treatment + The target engine speed signal is subjected to slope control treatment" was proposed and verified by simulation. The simulation results show that the coordination control strategy can effectively improve the tracking of the optimal fuel economy curve, improve the fuel economy 
performance of the engine, and improve the accuracy and stability of dynamic condition control.

\section{REFERENCES}

[1] Xiang Changle, Han Lijin, et al. An Analysis on the Characteristics of Power-split and Coupling Mechanism in Series/Parallel HEV[J]. Automotive Engineering, 2010, 32(3):183-187.

[2] Xiang Changle, Wu Yang, et al. Electric Power Coordinated Control Strategy of Dual-mode Electro-mechanical Transmission System[J]. Journal of Harbin Institute of Technology, 2017, 49(1):120-125.

[3] Chau K T, Wong Y S. Overview of Power Management in Hybrid Electric Vehicles[J]. Energy Conversion \& Management, 2002, 43(15):1953-1968.

[4] Borhan H, Vahidi A, Phillips A M, et al. MPC-Based Energy Management of a Power-Split Hybrid Electric Vehicle[J]. IEEE Transactions on Control Systems Technology, 2012, 20(3):593-603.

[5] Qin Datong, Peng Zhiyuan, et al. Dynamic Energy Management Strategy of HEV Based on Driving Pattern Recognition[J]. Chinese Journal of Mechanical Engineering, 2014, 25(11):1550-1555.

[6] Wang Lei, Zhang Yong, et al. Development of the Hybrid Control Unit for a Hybrid Electric Bus[J]. Automotive Engineering, 2012, 34(12):1065-1071.

[7] Luo Y, Serrani A, Yurkovich S, et al. Model-predictive Dynamic Control Allocation Scheme for Reentry Vehicles[J]. Journal of Guidance Control \& Dynamics, 2015, 30(1):100-113.

[8] Yan Yunbing, Yan Fuwu, et al. Experimental Research on Dynamic Coordinated Control for PHEV[J]. Journal of Highway and Transportation Research and Development, 2010, 27(1):126-131.

[9] Zhao Zhiguo, Dai Xianjun, et al. Coordinated Control of Driving Mode Switching of Compound Power-Split Hybrid Electric Car[J]. Automotive Engineering,2015(3):260-265.

[10] Wang Weida, Liu Hui, et al. Research on the Dynamic Power Control Strategy of the Two-mode Electro-mechanical Variable Transmission[J]. Journal of mechanical engineering, 2015, 51(12):101-109. 